\title{
Routinedaten - Wer hat die Deutungshoheit?
}

\author{
Routine Data in Psychiatry - Who has the Interpretative Power?
}

Autor

Institut
Hans Joachim Salize

Leiter Arbeitsgruppe Versorgungsforschung, Zentralinstitut für Seelische Gesundheit, Medizinische Fakultät Mannheim, Universität Heidelberg

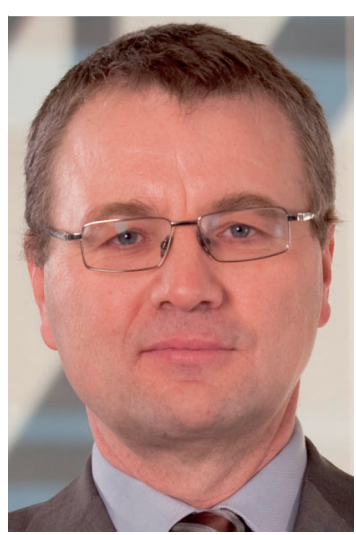

Prof. Dr. Hans Joachim Salize

Bibliografie

DOI http://dx.doi.org/

10.1055/s-0033-1343264

Psychiat Prax 2013; 40:

241-243

(c) Georg Thieme Verlag KG Stuttgart · New York

ISSN 0303-4259

Korrespondenzadresse

Prof.(apl) Dr.

Hans Joachim Salize

Leiter Arbeitsgruppe Versorgungsforschung,

Zentralinstitut für Seelische Gesundheit, Medizinische

Fakultät Mannheim,

Universität Heidelberg $\mathrm{J} 5$

68159 Mannheim

hans-joachim.salize@zi-

mannheim.de
Daten sind der Roh- und Treibstoff der Informationsgesellschaft, ähnlich wie es Erdöl im 20.Jahrhundert und Kohle während der Industrialisierung war. Spätestens seit der flächendeckenden Einführung von mobilen elektronischen Endgeräten ist die Verfügbarkeit und Interpretationshoheit über telematische Informationen und Daten die Bedingung für politische und ökonomische Dominanz. Die exponentielle Wachstumsdynamik, mit der elektronische Daten anfallen und verarbeitet werden können, ist dabei nicht nur der entscheidende gesellschaftspolitische Machtfaktor unserer Zeit, sondern viel weitreichender noch prägt diese Dynamik immer stärker die Art und Weise, wie die Welt wahrgenommen und interpretiert wird.

Dieser massive Einfluss auf Perzeption und Kognition tangiert unmittelbar die Sphäre der Psychiatrie. Diese Perspektive wird jedoch innerpsychiatrisch weitgehend ignoriert. Die Psychiatrie reagiert bisher klinisch wie wissenschaftlich sehr zurückhaltend auf die Auswirkungen, die soziale Netzwerke, die permanente Lokalisier-, Erreichund Kontrollierbarkeit von Individuen oder die zunehmende Beschneidung der Privatsphäre auf die psychische Gesundheit der Bevölkerung haben. Allenfalls solche zarten Versorgungsansätze wie für Internetsucht, Cyber-Mobbing oder Stalking etc. greifen diese Phänomene direkt auf.

Die psychiatrische Perspektive hinsichtlich des wachsenden Datenanfalls ist in der Regel eine ganz andere und im Vergleich zur angerissenen Problematik eine antiquierte. In der Regel assoziiert man innerpsychiatrisch beim Stichwort Routinedaten v.a. die zunehmende und zeitraubende Verpflichtung, im klinischen und therapeutischen Alltag Daten und Informationen zu dokumentieren, deren Nutzwert sich dem zur Dokumentation Verpflichteten meist verschließt. Dies dürfte die überwiegende Sichtweise der Versorgungspraktiker sein, wobei es sicherlich auch innerpsychiatrische Gruppen mit einem spezifischeren Routine- datenbewusstsein und -interesse gibt. Dies sind z.B. Administratoren, Controller oder epidemiologische und Versorgungsforscher. Auch die klinische oder Grundlagenforschung in der Psychiatrie hat ein vitales Dateninteresse, jedoch handelt es sich bei diesen Daten um hochspezielle und für den jeweiligen Untersuchungszweck eigens generierte Spezialinformationen, die sich grundlegend von dem in der Versorgungspraxis anfallenden Routinedatenkonvolut unterscheiden.

Lässt man die klinische und Grundlagenforschung beiseite, kann man die beiden traditionellen psychiatrischen Perspektiven auf Routinedaten als eine administrativ-abrechnungstechnische und eine (versorgungs-)epidemiologische Sichtweise charakterisieren. Beide Perspektiven haben unterschiedliche Bedeutung, die sich an Struktur, Verfügbarkeit und Qualität der verfügbaren psychiatrischen Routinedaten ablesen lässt. Die überwiegende Mehrzahl psychiatrischer Routinedatensammlungen ist finanz- oder vergütungstechnisch motiviert und strukturiert. Dies trifft auf sämtliche Dateien der gesetzlichen Krankenoder Rentenversicherung zu, eingeschlossen die Datensammlungen der Kassenärztlichen Vereinigung. Auch der (einzige) bundesweit einheitliche Datensatz in der stationären Krankenversorgung, der gemäß §21 des Krankenhausentgeltgesetzes (KHEntgG) von allen unter das KHEntgG fallenden Krankenhäusern jährlich an das Institut für das Entgeltsystem im Krankenhaus (InEK) zu liefern ist, offenbart Zweck und Ausrichtung der Daten bereits durch die Namensgebung der gesetzlichen Grundlage sowie des zu beliefernden Instituts.

Das Interesse, epidemiologische Evidenz oder Erkenntnisse für Versorgungssteuerung und -optimierung zu gewinnen, tritt gegenüber abrechnungs- und verteilungstechnischen Zielstellungen deutlich in den Hintergrund. Es existiert derzeit keine bundesweite einrichtungs- oder gar sektorenübergreifende Datensammlung, die das 
psychiatrische Versorgungsgeschehen unter primär epidemiologischen oder Aspekten der Versorgungseffektivität abbilden würde und als Instrument zur einrichtungs- oder sektorübergreifenden Versorgungssteuerung dienen könnte.

Dies müsste nicht so sein. Im stationärpsychiatrischen Bereich gab es immer wieder Versuche, eine bundesweit einheitliche Basisdokumentation psychiatrischer Krankenhausfälle mit relevanten klinischen Daten und Indikatoren des Behandlungsergebnisses zu etablieren. Die flächendeckende Einführung ist letztlich immer am mangelnden Engagement derjenigen, die die Daten liefern müssten, gescheitert. Allenfalls in begrenzt lokalen oder regionalen Verbünden haben sich psychiatrische Kliniken zusammengefunden, die ihre Behandlungsepisoden einheitlich dokumentieren und diese Daten zusammenführen. Dies allerdings meist auch nur dann, wenn sie verwaltungstechnisch sowieso eine Einheit bilden.

Die methodischen Probleme einer Dokumentation von Routinedaten, die das gesamte stationäre, ambulante und rehabilitative Leistungs- und Einrichtungsspektrum der Gemeindepsychiatrischen Verbünde in einem vereinheitlichten und gleichzeitig detaillierten Datenset abbildet, sind prinzipiell schon lange gelöst, z.B. durch Projekte in Sachsen oder Baden-Württemberg [1, 2]. Aber auch diese Initiativen hatten angesichts der Hemmnisse der Implementierung einheitlicher Systeme in Einzelsektoren keine Chance einer flächendeckenden Einführung, obwohl sie neben Struktur- und Prozessdaten auch aussagekräftige Indikatoren des Behandlungserfolgs liefern könnten und sich bestens zur Versorgungssteuerung eignen würden.

Neben der Zustimmung und Mitwirkung des gesamten fragmentierten Einrichtungsspektrums in der Psychiatrie ist die größte Implementierungshürde stets der Dokumentationsaufwand, der zusätzlich zur täglichen Versorgungsroutine geleistet werden muss und nicht gesondert finanziert wird. Dies vereitelt die überregionale Anwendung eines jeden noch so gut erarbeiteten Ansatzes. Der Dokumentationsaufwand wächst proportional zur Detailliertheit und Aussagekraft der Daten. Die Erfassung geht immer von der Pflege- oder Therapiezeit ab, wobei der generelle Trend, im klinischen Sektor Pflegeleistungen zugunsten der Ausweitung des Qualitätsmanagements zu beschneiden, die Sache nicht besser macht.

Das Ergebnis dieses Dilemmas ist eine strategische Defensive des Faches Psychiatrie hinsichtlich der Verfügbarkeit und Deutungshoheit über strukturierte Informationen ihres eigenen Leistungsspektrums. Dies führt zu solch absurden Situationen, dass die Psychiatrie noch nicht einmal Auskunft über Mengenverhältnisse in ihren sensibelsten Bereichen geben kann, weil sie darüber Buch zu führen nicht in der Lage ist. Dies gilt z. B. für die Zahl der jährlichen Zwangseinweisungen in psychiatrische Krankenhäuser oder Abteilungen. Will man dies wissen, müssen außerpsychiatrische Behörden konsultiert werden, wie z.B. die Amtsgerichte, die sehr wohl solche Informationen sammeln und veröffentlichen - allerdings strukturiert nach ihren eigenen und nicht nach psychiatrischen Anforderungen [3]. Die Nachteile und Peinlichkeiten solcher Informationsmängel sind evident, und wenn auch nur in der öffentlichen Debatte, in der die Anwendung von Zwang immer noch zu großen Teilen das Ansehen der Psychiatrie bestimmt.

In der sich gerade vollziehenden Reform des Entgeltsystems in der stationären Psychiatrie wird die mangelnde Informationsbasis und Argumentationsmacht erst richtig schmerzhaft. Zu keinem Zeitpunkt des Verfahrens waren das Fach und seine Interessenvertreter in der Lage, mit evidenzbasierten Argumenten in die
Diskussion einzugreifen oder auf der Basis eigener Informationsquellen erarbeitete Alternativmodelle der Finanzierung anzubieten.

Es gibt Versuche, dies zu ändern, wie z.B. in der von der DGPPN und anderen getragenen VIPP-Initative („Versorgungsrelevante Indikatoren in Psychiatrie und Psychotherapie“). Hierbei soll ab dem laufenden Jahr ein adaptierter Teil des oben genannten §21 KHEntgG-Datensatz zur Generierung von versorgungsepidemiologischen Erkenntnissen genutzt werden [4]. Das Mitwirken an dieser Datenbasis ist jedoch freiwillig und bedarf eines datenschutzrechtlich unbedenklichen Verfahrens, das mittlerweile etabliert ist. Die Bewerbung der Initiative bei psychiatrischen Kliniken und Abteilungen läuft gegenwärtig bundesweit. Solche Bemühungen zur Errichtung einer eigenen Informationsbasis sind unverzichtbar. Inwieweit sie sich bewähren, muss die Zeit zeigen. Solange sie jedoch auf abrechnungstechnisch strukturierten und auf einzelne Einrichtungstypen beschränkten Datensätzen aufbauen, werden Erkenntnisse hinsichtlich einrichtungsübergreifender Versorgungsmuster oder Ergebnisindikatoren eingeschränkt bleiben. Daran ändern auch alle Bemühungen nichts, den Erkenntnisgehalt von fragmentierten psychiatrischen Routinedaten hinsichtlich der Ableitbarkeit von Qualitätsindikatoren zu nutzen und durch entsprechende Analysestrategien zu erweitern $[5,6]$. Allein schon die geringe Validität diagnosebezogener Daten und Verfahren in administrativen Datensätzen ist ein methodisch überaus gravierendes und kaum lösbares Problem für versorgungsepidemiologische Schlussfolgerungen.

Selbst wenn es auf diese oder andere Weise gelänge, eine eigene Daten- und Interpretationsbasis zu gewinnen, dürfte dies die strategische Defensive der Psychiatrie hinsichtlich der Deutungshoheit über das eigene Fach kaum verändern. Mit der ungeheuren Geschwindigkeit, in der sich die Möglichkeiten zur Datenschürfung und -verarbeitung verbessern, werden die wahren Routinedatenschlachten längst woanders geschlagen. Es wäre naiv anzunehmen, dass bspw. die Pharmaindustrie nicht nach dem Muster von Google, Facebook oder Apple mit Methoden des Datenminings die ihnen zugänglichen Datenquellen kombiniert und hinsichtlich des Konsumentenverhaltens (d.h. das der Verschreiber und der Patienten) durchkämmt und damit Marketingstrategien optimiert. Soziale und Praxisnetzwerke werden dabei kaum außen vor bleiben. Solche sog. kliodynamischen Techniken werden für die gesamten produzierenden und Handel treibenden Sektoren in den nächsten Jahren überlebenswichtig. Inwieweit der Daten- und Persönlichkeitsschutz, der es innerpsychiatrisch zu Recht seit jeher genau nimmt, mit solchen Ansätzen Schritt halten kann, ist zweifelhaft. Die Psychiatrie selbst kann angesichts ihrer oben beschriebenen Mühen von solchen Verfahren, die weit über die Möglichkeiten eines noch so gut funktionierenden Fallregisters hinausgehen, nur träumen - ganz abgesehen davon, dass sie in der Psychiatrie (und nicht nur dort) ethisch mehr als fragwürdig wären.

Damit liegt das eigentliche Problem, das die Psychiatrie mit Routinedaten bekommen wird bzw. schon jetzt hat, nicht in der Frage, ob sie gewillt ist, solche zu produzieren oder in der Lage ist, die Deutungshoheit darüber zu erlangen, sondern es ist psychopathologischer Natur. Die Preisgabe, Abschöpfung und akzelerierte Kommerzialisierung persönlichster Informationen sowie die Verfüg- und Nachverfolgbarkeit aller Individuen wird in den nächsten Jahren ein Ausmaß annehmen, das wir gegenwärtig noch kaum zu ahnen imstande sind. Dies wird die Krankheitsmuster des 21. Jahrhunderts in ähnlicher Weise prägen wie es Tuberkulose, Staublunge oder andere Berufskrankheiten in den in- 
dustriellen Zentren des 19. Jahrhunderts getan haben. Nur dass es diesmal nicht nur Industriearbeiter oder andere selektive Gruppen trifft, sondern die gesamte Bevölkerung. Die alljährlichen Krankenkassenreports mit ihren rapide steigenden Sucht-, Burn-out- oder Depressionsraten berichten bereits schon jetzt nichts anderes.

Damit sind Routinedaten für die Psychiatrie nicht mehr länger nur ein lästiges administratives Problem, das irgendwie in die Abläufe psychiatrischer Einrichtungen integriert werden muss, sondern sie gewinnen den Charakter einer epidemiologisch hoch relevanten psychopathologischen Noxe. Es wird das Bild und die Leistungsbilanz der Psychiatrie der Zukunft entscheidend prägen, ob sie hierauf eine Antwort zu finden vermag.

\section{Literatur}

1 Salize HJ, Bullenkamp J, Alscher I et al. Qualitätssicherung und Dokumentation im Gemeindepsychiatrischen Verbund (GPV) - ein standardisiertes Verfahren für die regionale Versorgungsdokumentation und -steuerung. Psychiat Prax 2000; 27: $92-98$

2 Kallert TW, Schützwohl M, Leiße $M$ et al. Dokumentationssystem für den komplementären psychiatrischen Versorgungsbereich. Entwicklung und Erprobung im Freistaat Sachsen. Psychiat Prax 2000; 27: $86-91$

3 Salize HJ, Spengler A, Dressing H. Zwangseinweisungen psychisch Kranker - wie spezifisch sind die Unterschiede in den Bundesländern. Psychiat Prax 2007; 34 (Suppl. 02): S196-S202

4 Godemann F, Falkai P, Hauth I et al. Pauschalierendes Entgeltsystfem in der Psychiatrie und Psychosomatik. Begleitforschung - quo vadis? Nervenarzt 2013: online first

5 Gaebel W, Zilasek J, Kotwitz S. Die Nutzung von Rotuinedaten für die psychiatrische und psychosomatische Versorgungsforschung. Psychiatrie 2011; 8: 23-33

6 Zilasek J, Großimlinghaus I, Janssen B et al. Die Rolle von Qualitätsindikatoren in der psychiatrischen Qualitätssicherung und aktueller Stand der Entwicklung von Qualitätsindikatoren. Psychiatrie 2012; 9: 46-52 